\title{
The embryonic expression pattern of a second, hitherto unrecognized, paralog of the pair-rule gene sloppy-paired in the beetle Tribolium castaneum
}

\author{
Ralf Janssen ${ }^{1}$ (iD \\ Received: 17 February 2020 / Accepted: 11 May 2020 / Published online: 20 May 2020 \\ (C) The Author(s) 2020
}

\begin{abstract}
In the fly Drosophila melanogaster, a hierarchic segmentation gene cascade patterns the anterior-posterior body axis of the developing embryo. Within this cascade, the pair-rule genes (PRGs) transform the more uniform patterning of the higher-level genes into a metameric pattern that first represents double-segmental units, and then, in a second step, represents a true segmental pattern. Within the PRG network, primary PRGs regulate secondary PRGs that are directly involved in the regulation of the next lower level, the segment-polarity genes (SPGs). While the complement of primary PRGs is different in Drosophila and the beetle Tribolium, another arthropod model organism, both paired (prd) and sloppy-paired (slp), acts as secondary PRGs. In earlier studies, the interaction of PRGs and the role of the single slp ortholog in Tribolium have been investigated in some detail revealing conserved and diverged aspects of PRG function. In this study, I present the identification and the analysis of embryonic expression patterns of a second slp gene (called $s l p 2$ ) in Tribolium. While the previously identified gene, slp, is expressed in a typical PRG pattern, expression of $\operatorname{slp} 2$ is more similar to that of the downstream-acting SPGs, and shows expression similarities to slp2 in Drosophila. The previously reported differences between the function of slp in Drosophila and Tribolium may partially account for the function of the newly identified second slp paralog in Tribolium, and it may therefore be advised to conduct further studies on PRG function in the beetle.
\end{abstract}

Keywords Sloppy-paired $\cdot$ Slp $\cdot$ FoxG $\cdot$ Segmentation $\cdot$ Pair-rule $\cdot$ Segmental patterning $\cdot$ Forkhead

\section{Introduction}

In the dipteran fly Drosophila melanogaster, a hierarchic segmentation gene cascade almost synchronously controls the patterning of the early embryo along the anterior-posterior (AP) body axis into a defined number of single segments (reviewed in e.g. Akam 1987, Davis and Patel 2003). First, maternally provided factors form anterior to posterior and posterior to anterior morphogen gradients, that then regulate the zygotically

Communicated by Nico Posnien

Electronic supplementary material The online version of this article (https://doi.org/10.1007/s00427-020-00660-x) contains supplementary material, which is available to authorized users.

Ralf Janssen

ralf.janssen@geo.uu.se

1 Department of Earth Sciences, Palaeobiology, Uppsala University, Villavägen 16, 75236 Uppsala, Sweden expressed gap genes (GGs) (e.g. Fröhnhofer and NüssleinVolhard 1986). The GGs, which are expressed in broad overlapping domains along the AP axis of the developing embryo, regulate another group of zygotically expressed genes, the socalled pair-rule genes (PRGs). PRGs are typically expressed in the form of seven transversal stripes corresponding to alternating segment primordia (e.g. Jäckle et al. 1992). For most of the PRGs, these primary expression patterns change into a segmental pattern. Within the PRG network, the so-called primary PRGs such as even-skipped (eve) and hairy (h) act upstream of the so-called secondary PRGs, which in Drosophila are sloppy-paired (slp) and paired (prd) (reviewed in e.g. Clark 2017). In a complex combinatorial interaction, the PRGs then regulate the activity of the segment-polarity genes (SPGs), which act to maintain the parasegment borders, and define the polarity of each segment (e.g. Ingham et al. 1988, Cadigan et al. 1994a, recently reviewed in Clark et al. 2019).

This mode of segment formation, in which all segments are patterned synchronously at the blastoderm stage, is derived, and most other insects, as well as all other arthropods, only 
form their most anterior segments in a similar way (reviewed in Peel 2004). Posterior segments, however, are added from a posterior segment addition zone (SAZ). In most cases, single segmental units are added from the SAZ (e.g. Hughes and Kaufman 2002; Schoppmeier and Damen 2005; Janssen et al. 2011), but in some arthropods, all, or a number of segments, are added (or patterned) with a double-segment periodicity (e.g. Binner and Sander 1997; Dearden et al. 2002; Chipman et al. 2004; Erezyilmaz et al. 2009; Janssen et al. 2012; El-Sherif et al. 2012; Sarrazin et al. 2012). This latter mechanism is reminiscent of the initial expression of the PRGs in Drosophila and their pair-rule function. While it is still unclear if pair-rule patterning (and function) is a conserved ancestral trait of arthropod segmentation, it appears likely that the PRGs in general are involved in segmentation, as evident from functional studies (e.g. Liu and Kaufman 2005; Mito et al. 2007; Rosenberg et al. 2015; Xiang et al. 2017; Auman and Chipman 2018), and the analysis of gene expression patterns (e.g. Damen et al. 2000, 2005; Dearden et al. 2002; Hughes and Kaufman 2002; Chipman and Akam 2008; Janssen et al. 2011; Green and Akam 2013; Schönauer et al. 2016).

Compared with Drosophila, the beetle Tribolium castaneum displays a more conservative mode of development: The anterior segments are formed from the blastoderm, but posterior segments are added sequentially. In both the blastoderm that give rise to the anterior segments, and the posteriorly added segments, a clock-like mechanism including the function of PRGs appears to be involved. This mechanism generates/patterns segments with a double-segment periodicity (Choe et al. 2006; El-Sherif et al. 2012; Sarrazin et al. 2012), although the last-formed posterior segments indeed may be patterned one by one, at least on the level of SPGs (Janssen 2014). Generally, PRG orthologs have been in the focus of unravelling the segmentation mechanisms in Tribolium (e.g. Sommer and Tautz 1993, Brown et al. 1994, 1997, Maderspacher et al. 1998, Schröder et al. 2000, Eckert et al. 2004, Aranda et al. 2008, Bolognesi et al. 2009, Choe and Brown 2007, 2009, Peel et al. 2013, El-Sherif et al. 2014), and one of these genes of interest is the sloppy-paired (slp) gene (Choe et al. 2006; Choe and Brown 2007).

The PRG ortholog sloppy-paired (slp) (alternative name Fox $G$ ) encodes a forkhead-box containing transcription factor, that acts as a secondary PRG in Drosophila, where it exists in the form of two paralogs (slp1 and slp2) (Häcker et al. 1992; Grossniklaus et al. 1992). Both paralogs function directly on the SPGs and also act as SPGs. In this function, they are involved in the maintenance of parasegmental boundaries and segment polarity (Cadigan et al. 1994a). In accordance with this dual role, slp paralogs are first expressed in the form of seven transverse segmental stripes (PRG pattern), and later in the form of 14 stripes (SPG pattern) (Grossniklaus et al. 1992).
Similar to the two Drosophila paralogs, the single described Tribolium slp gene appears to function downstream of a regime of primary PRGs (Choe et al. 2006), and is involved in the regulation of SPGs, and thus in segmentation, albeit in a slightly different way than in Drosophila (Choe and Brown 2007, 2009).

In this paper, I present the discovery of a second, hitherto unrecognized, paralog of sloppy-paired in Tribolium. Its embryonic expression pattern suggests a potential role in segmentation, either downstream and/or in parallel with the other Tribolium PRG orthologs, including the earlier-described Tribolium slp gene (Choe et al. 2006). The presence of a second $s l p$ gene, designated as $\operatorname{sip2}$, may have implications for the interpretation of earlier research conducted on PRG patterning and the gene regulatory network that governs segmentation in Tribolium.

\section{Methods}

\section{Tribolium husbandry and preparing embryos}

The used specimens of Tribolium castaneum stem from the culture in Göttingen/Germany. A colony of this strain was established in Uppsala, following the suggestions made in "The Beetle book" (link: http://wwwuser.gwdg.de/ $\sim$ gbucher1/tribolium-castaneum-beetle-book1.pdf). Embryos were collected and prepared for subsequent in situ hybridization experiments as per Schinko et al. (2009).

\section{Extraction of total RNA, cDNA synthesis, gene cloning and whole mount in situ hybridization}

Total RNA was isolated from complete embryos of mixed developmental stages using TRIZOL (Invitrogen). Total RNA was reverse transcribed into cDNA using the SuperScript First Strand kit (Invitrogen). Gene fragments were isolated by RT-PCR with gene-specific primers. Primer sequences are $s l p$ forward: GGTGAAAAGGGAAG AAACGA, slp_backward: AGATCACCGTCACT GGTTTA, slp2_forward: GGCAGAGAGCAAAG AAACCG and slp2_backward: ACAGTGACAGGTTT GAGGAG). Gene fragments were ligated into the PCRII vector (Invitrogen) and sequenced on an ABI3730XL automatic sequencer (Macrogen, Seoul, South Korea). Unique gene identifiers are listed in Supplementary File 1. Monochromatic in situ hybridizations were performed using the standard protocol provided in Janssen et al. (2018). Dichromatic in situ was performed as described in Janssen et al. (2008). Monochromatic in situs were performed with digoxigenin (DIG)-labelled probes; for the second staining in dichromatic in situs, a fluorescein-labelled second probe was used. First, the digoxigenin-labelled probes were detected 
Fig. 1 Phylogenetic analysis. Sloppy-paired genes, including monophyletic group with high support (red lines), that is separated from the other Drosophila Fox genes. The derived sequence of Drosophila FoxO serves as an outgroup. Positions of the two paralogs of Tribolium sloppyarrows. The red arrow points to the newly-discovered Slp2. Species abbreviations: Cs, Cupiennius salei (Chelicerata: Aranea); Dm, Drosophila melanogaster (Hexapoda: Diptera); Ek, Euperipatoides kanangrensis (Onychophora); $\mathrm{Gm}$, Glomeris marginata (Myriapoda: Diplopoda), Tc, Tribolium castaneum (Hexapoda: Coleoptera) the newly identified Slp2, form a paired sequences are marked with

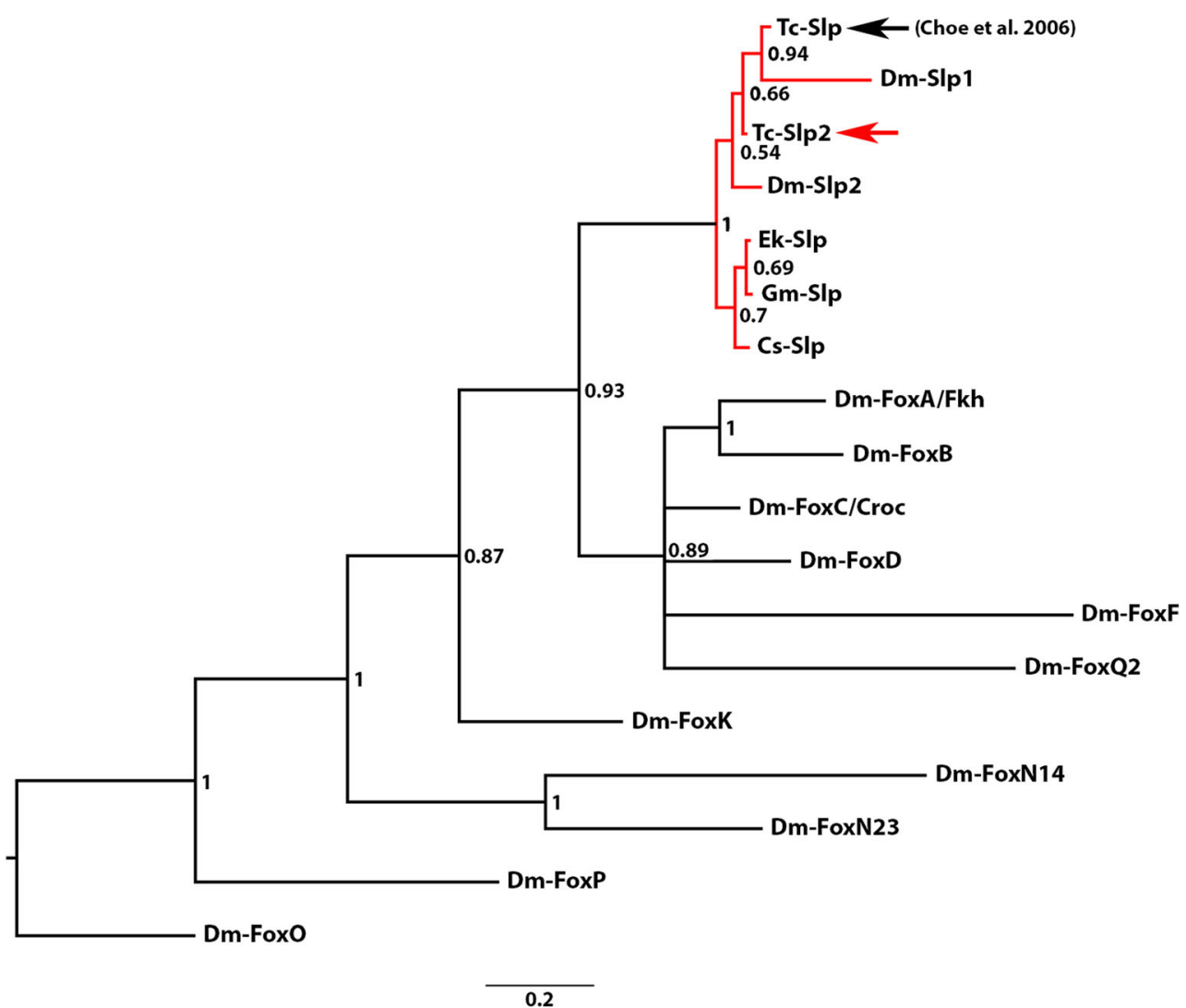

with BMPurple (Roche), giving rise to a blue signal. After that, alkaline phosphatase on the DIG-labelled antibodies was inactivated with $0.1 \mathrm{M}$ glycine, $\mathrm{pH}=2.0$ (2-min incubation at room temperature), and the second probe was detected with SIGMAFAST Fast Red TR/Naphtol AS-Mx (Sigma), giving rise to a red signal.

\section{Identification of potential paralogs of sloppy-paired and phylogenetic analysis}

Paralogs of Tribolium sloppy-paired (slp) were identified performing reciprocal BLAST searches (BlastX and BlastP) against the published genomic sequences of the beetle Tribolium using the Drosophila paralogs slp1 and slp2, and the previously identified Tribolium slp gene (Choe et al. 2006) as baits.

Amino acid sequences of the forkhead domains were aligned using T-Coffee (Notredame et al. 2000) using default parameters in MacVector v12.6.0 (MacVector, Inc., Cary, NC). A Bayesian phylogenetic analysis was performed using MrBayes (Huelsenbeck and Ronquist 2001) with a fixed WAG amino acid substitution model with gammadistributed rate variation across sites (with four rate categories), unconstrained exponential prior probability distribution on branch lengths, and exponential prior for the gamma shape parameters for among-site rate variation. Tree topology was calculated applying 200,000 cycles for the MetropolisCoupled Markov Chain Monte Carlo (MCMCMC) analysis (four chains; chain-heating temperature of 0.2). Markov chains were sampled every 200 cycles. Default settings were used, defining $25 \%$ of the samples as burn-in information. Clade support was calculated with posterior probabilities in MrBayes. Unique identifiers of all used sequences are summarized in Supplementary File 1.

\section{Data documentation}

Pictures of in situ-stained embryos were taken with a Leica DC490 digital camera mounted onto a MZ-FLIII Leica dissection stereo-loupe. Linear adjustments were made on contrast and brightness using the image-processing software Adobe Photoshop CC 2018 for Apple Macintosh (Adobe Systems Inc.)

\section{Results}

\section{Identification of Tribolium sloppy-paired genes}

The performed reciprocal BLAST search identified two genes with high sequence similarity to both Drosophila melanogaster sloppy-paired genes (slp1 and slp2) (Häcker 


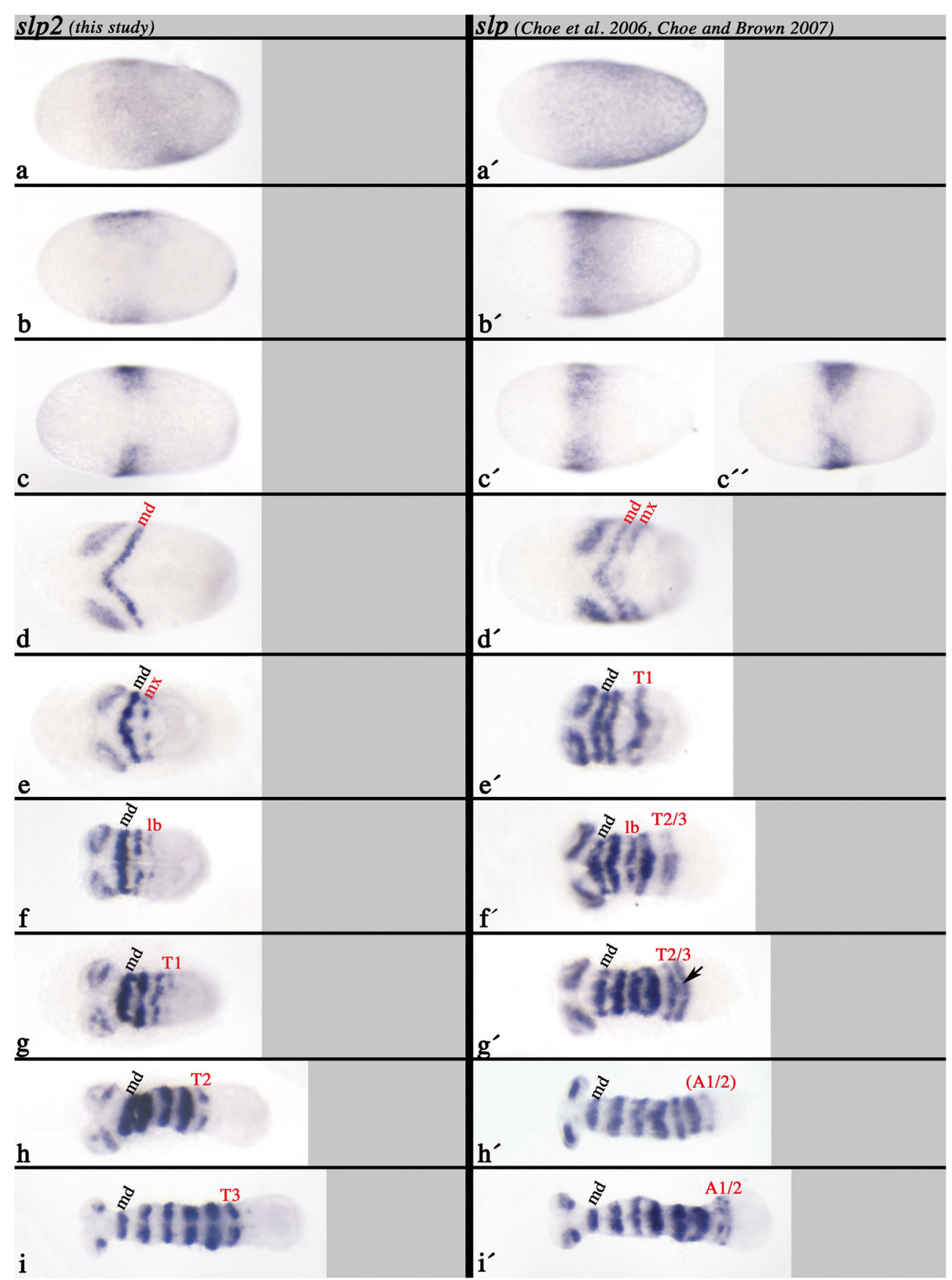

et al. 1992; Grossniklaus et al. 1992), the single previously described Tribolium sloppy-paired gene (slp) (Choe et al. 2006), and confirmed sloppy-paired orthologs from other arthropods (e.g. Damen et al. 2005, Liu and Patel 2010, Janssen et al. 2011, Green and Akam 2013, Auman and Chipman 2018) and an onychophoran (Janssen and Budd 2013).

Phylogenetic analysis shows that these two Tribolium genes cluster with panarthropod sloppy-paired orthologs and form a well-supported monophyletic group (Fig. 1). Tribolium Slp clusters with high confidence with Drosophila Sloppypaired1 (Slp1), and it seems clear that those two form an orthology-pair. The newly identified Tribolium Slp2 and Drosophila Slp2 do not form a sister-gene relationship in the phylogenetic analysis. The posterior probability values, however, that separate Tribolium and Drosophila Slp2 from each another are very low. Therefore, it is possible that the two designated slp2 genes are in fact the result of a duplication at the lineage leading to Tribolium + Drosophila, and thus could represent paralogs. Note that the distribution of Slp proteins does not represent our current understanding of arthropod relationships. 
Fig. 2 a Expression of $s l p$ (Choe et al. 2006) and the newly identified slp2-EARLY STAGES. In all panels, anterior is to the left, ventral views. a-i Expression of $\operatorname{slp} 2$. $\mathbf{a}^{\prime}-\mathbf{i}^{\prime}$ expression of $s l p$ in comparable stages (as a-i). Newly formed segments (expression) are indicated in red letters. a/a' Early Blastoderm stage. Expression in the posterior $3 / 5$ of the embryo. b Later blastoderm stage. Expression retracts from the posterior of the embryo. $\mathbf{c} / \mathbf{c}^{\prime} / \mathbf{c}^{\prime \prime}$ Late blastoderm stage. slp2 is expressed in two domains separated by a ventral gap. slp is first expressed in a gapgene like domain, that later splits ventrally $\left(\mathbf{c}^{\prime \prime}\right)$. $\mathbf{d} / \mathbf{d}^{\prime}$ Gastrulation stage. The gap-gene like domain is split into expression in the future antennal and ocular region. The mandibulary stripe (md) of $\operatorname{slp} 2$ has formed; md and maxillary $(\mathrm{mx})$ stripes of $s l p$ have formed. Note the simultaneous appearance of two stripes of slp. e/e' $\operatorname{slp} 2$ appears in the mx. slp appears in the first thoracic segment (T1). f// $\mathbf{f}^{\prime}$ slp 2 appears in the labial segment (lb). slp appears in lb., and in a broad domain representing T2 and T3. g/g' slp appears in T1. slp2 expression in T2 and T3 splits into two separate stripes. The arrow points to remnants of expression corresponding to the earlier seen broad domain. $\mathbf{h} / \mathbf{h}^{\prime} \operatorname{slp} 2$ appears in T2. slp appears in a broad domain representing the primordia of the first two abdominal segments (A1 and A2). i/i' slp2 appears in T3. slp expression in A1 and A2 forms separate stripes, b Expression of slp (Choe et al. 2006) and the newly identified $\operatorname{slp2}$-LATER STAGES. In all panels, anterior is to the left, ventral views. $\mathbf{j}-\mathbf{s}$ Expression of $s l p 2$. $\mathbf{j}^{\prime}-\mathbf{s}^{\prime}$ Expression of $s l p$ in comparable stages (as $\mathbf{j}-\mathbf{s}$ ). Newly formed segments (expression) are indicated in red letters. $\mathbf{j} / \mathbf{j}^{\prime} \operatorname{slp} 2$ appears (weakly) in the intercalary segment (ic) and in A1. slp appears in ic and the primordia of A3 and A4. Note the space between this new stripe and the last-formed stripe in A4 (black bar). $\mathbf{k} / \mathbf{k}^{\prime}$ slp2 appears in A2 (and weakly in an anterior to posterior order) in A3. slp expression in $\mathrm{A} 3$ and $\mathrm{A} 4$ forms separate stripes. The arrow points to remnants of expression corresponding to the earlier seen broad domain. $\mathbf{1} / \mathbf{I}^{\prime} \operatorname{slp} 2$ appeared in A3 and (weakly) appears in A4. slp is expressed as two stripes representing A5 and A6. $\mathbf{m} / \mathbf{m}^{\prime}$ slp2 is in A5 and (weakly) appears in A6 and (very weakly) in A7. slp appears in the primordium of A8. Note the space between this new stripe and the last-formed stripe in A6 (black bar). $\mathbf{n} / \mathbf{n}^{\prime} \operatorname{slp} 2$ weakly appears in A8. slp appears in the primordium of A7 between the stripes in A6 and the primordium of A8. o/o' slp2 appeared in A8. slp appeared in A9 and A10. p/p' slp2 appeared in A9. slp is in A10. $\mathbf{q} / \mathbf{q}^{\prime}$ slp2 appeared in A10. slp appears in a domain posterior to A10 (asterisk). r/r' $\mathbf{r}^{\prime} \operatorname{slp} 2$ appears in a domain posterior to A10 (asterisk). s/s's slp2 and $s l p$ are expressed in identical segmental patterns

\section{Expression of the previously described Tribolium sloppy-paired (slp) in comparison with the newly identified second paralog, slp2}

The Tribolium slp gene has been identified in Choe et al. (2006), and its expression has been described in some detail in Choe and Brown (2007). Here, I present a more detailed description of $s l p$ in a series of consecutive developmental stages, revealing some additional aspects of its expression, and at the same time compare its expression with that of slp2 (Fig. 2).

At the blastoderm stage, both paralogs of slp are expressed in identical patterns in the complete embryo, except for its anterior cap that will later give rise to the serosa (Fig. 2a/a'). In a slightly later blastoderm stage, expression of both paralogs disappears from the posterior of the embryo resulting in a broad central domain of expression (Fig. 2b/b'). In contrast to slp (Fig. 2b'), expression of $\operatorname{slp} 2$ is weaker ventrally and finally fully disappears, resulting in two separate domains of expression in the following stage (Fig. 2c). The same ventral disappearance of expression happens with $s l p$, but at a slightly later stage (Fig. $2 \mathrm{c}^{\prime \prime}$ ). This is evident from the presence of an intermediate stage in which expression is visible in the form of a solid band (i.e. prior to ventral disappearance) (cf. Fig. $2 \mathrm{c}^{\prime} / \mathrm{c}^{\prime \prime}$ ). With the beginning of gastrulation (Fig. $2 \mathrm{~d} / \mathrm{d}^{\prime}$ ), two stripes of slp appear simultaneously and posterior to the anterior expression domain which is located in the head and likely contributes to the anlagen of the antennae and the eyes (see later developmental stages). The two new stripes correspond to the mandibular (md) and maxillary (mx) segment (Fig. 2d'). At the same time, only one stripe of slp2 appears in the anlage of the mandibles (md) (Fig. 2d). The next stripe of slp expression appears in the first thoracic segment (T1) (Fig. 2e'), while $s l p 2$ now appears in the $\mathrm{mx}$ (Fig. 2e). slp expression then appears in the labial (lb) segment and, in the form of a single broad domain, in the anlagen of the second and the third thoracic segments (T2 and T3) (Fig. $2 \mathrm{f}^{\prime}$ ). slp2, however, only appears newly in the $\mathrm{lb}$. (Fig. $2 \mathrm{f}^{\prime}$ ). The broad domain of $s l p$ in T2/T3 then separates into two stripes (Fig. $2 \mathrm{~g}^{\prime}$ ), while for $\operatorname{slp} 2$, the T1 stripe forms (Fig. 2g). Note the remnants of earlier expression (the broad domain) of $\operatorname{slp} 2$ between the now-forming two stripes (Fig. 2g', arrow; also see Fig. 2k'). Then another broad posterior domain of $s l p$ appears which is corresponding to the first two abdominal segments (A1/A2) (Fig. 2h'). At approximately the same time, expression of slp2 appears in T2 (Fig. 2h). As the posterior expression of slp splits into separate stripes in A1 and A2, the T3 stripe of slp2 appears (Fig. 2i/i'). At the following stage, two new domains of expression appear for both genes. The first is the "delayed" appearance in the intercalary segment (ic) $($ Fig. $2 \mathrm{j} / \mathrm{j}$ '). Additionally, another broad domain of slp appears representing the third and fourth abdominal segments (A3/A4) (Fig. 2j'), and, at this time, also the first abdominal stripe of $\operatorname{slp} 2$ appears (Fig. $2 \mathrm{j})$. This pattern of posterior stripe-addition and splitting (for $s l p$ ) is maintained in the following developmental stages: addition of A3/A4 (slp) and A2 (A3 weakly) (slp2) (Fig. 2k/k'), addition of A5/A6 (slp) and A3 (A4 weakly) (slp2) (Fig. 21/1'), addition of A7/A8 (slp) and A5 (A6 and A7 weakly) (slp2) (Fig. $2 \mathrm{~m} / \mathrm{m}^{\prime}$ ), splitting of A7/A8 (slp) and A6 (A7 and A8 weakly) (slp2) (Fig. 2n/ $\mathrm{n}^{\prime}$ ), addition of A9/A10 (slp) and A8 (slp2) (Fig. 2o/o'), splitting of A9 and A10 (slp) and addition of A9 (slp2) (Fig. $2 \mathrm{p} / \mathrm{p}^{\prime}$ ). Then, a single stripe of $s l p$ forms posterior to the A10-stripe (Fig. 2q'), and the A10 stripe of slp2 forms (Fig. 2q). As this last added stripe of slp becomes stronger, the same stripe of $\operatorname{slp} 2$ forms (Fig. $2 \mathrm{r} / \mathrm{r}^{\prime}$ and $\left.\mathrm{s} / \mathrm{s}^{\prime}\right)$. 


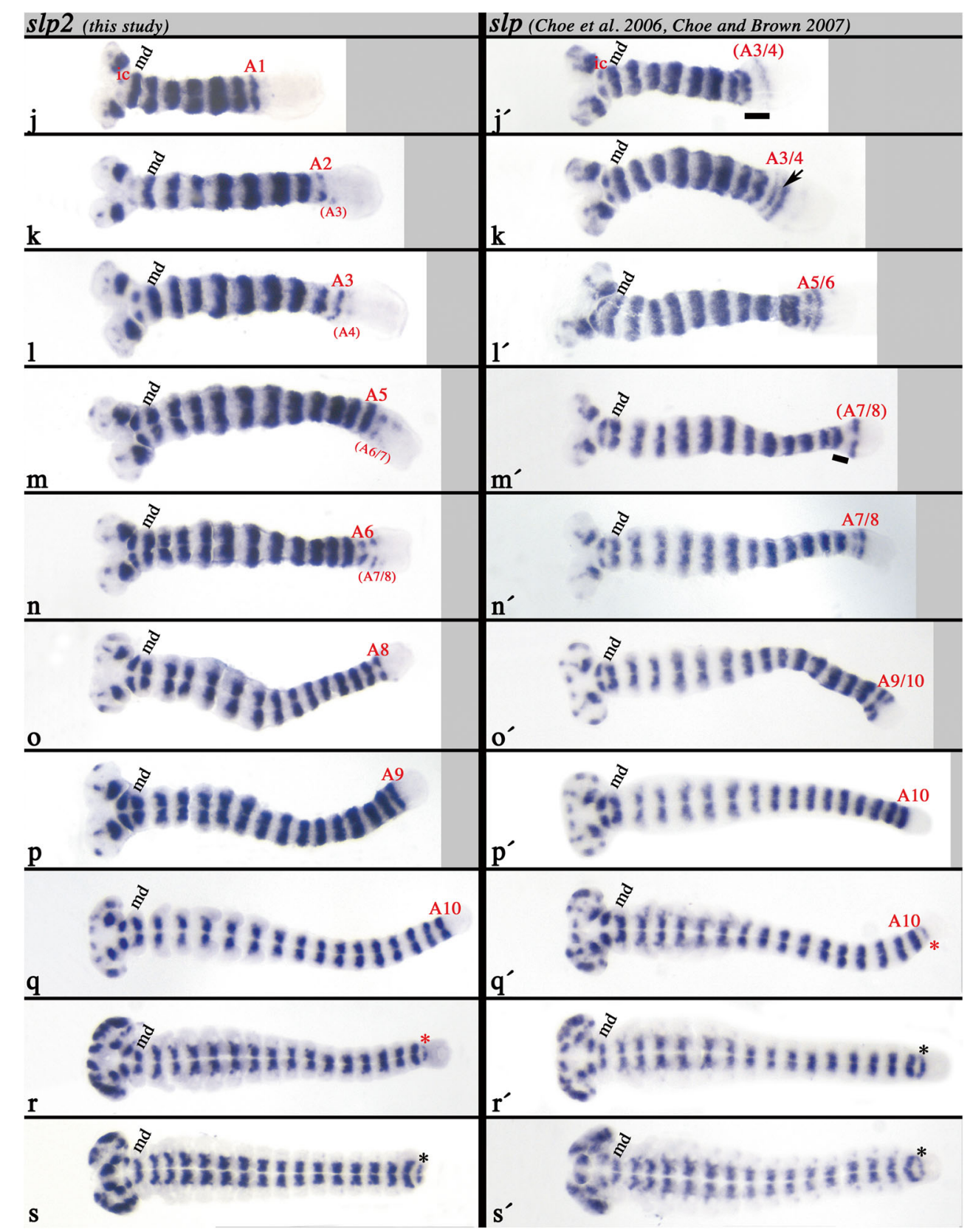

Fig. 2 (continued)

Expression of $s l p$ in the labrum appears at the stage depicted in Fig. 2o'. Expression of slp2 in the labrum, however, appears slightly later in the embryo shown in Fig. 2q.

In summary, segmental expression of $s l p$ appears in a doublesegmental pattern and involves either the splitting of an initial broad domain into two distinct stripes in adjacent segments, or intercalation of a secondary stripe anterior to the last-formed stripe. Stripes of $s l p 2$, however, appear in an anterior to posterior order with a single-segment periodicity. The segmental stripes of $s l p$ appear earlier than those of $s l p 2$ (Fig. 2).

The intra-segmental position of $s l p$ has been shown to be anteriorly adjacent to the expression of the SPG engrailed (en) (Choe and Brown 2007). Double expression of $s l p$ with the downstream target of En, hedgehog $(h h)$, corroborates this finding (Fig. 3a/b). The intrasegmental position of slp2 is almost identical with that of $s l p$ as revealed by co-expression with $h h$ (Fig. 3c/d). Double in situs with $\operatorname{slp}$ and $\operatorname{slp} 2$, however, reveal that the expression of $s l p$ extends somewhat more towards anterior than that of $\operatorname{slp} 2$ (Fig. 3e, f, g, h).

With respect to their temporal appearance during segment addition, the appearance of the segmental expression of $s l p$ predates the onset of $\operatorname{slp} 2$-expression in stripes (Figs. 2 and 3e, $f, g, h)$. 


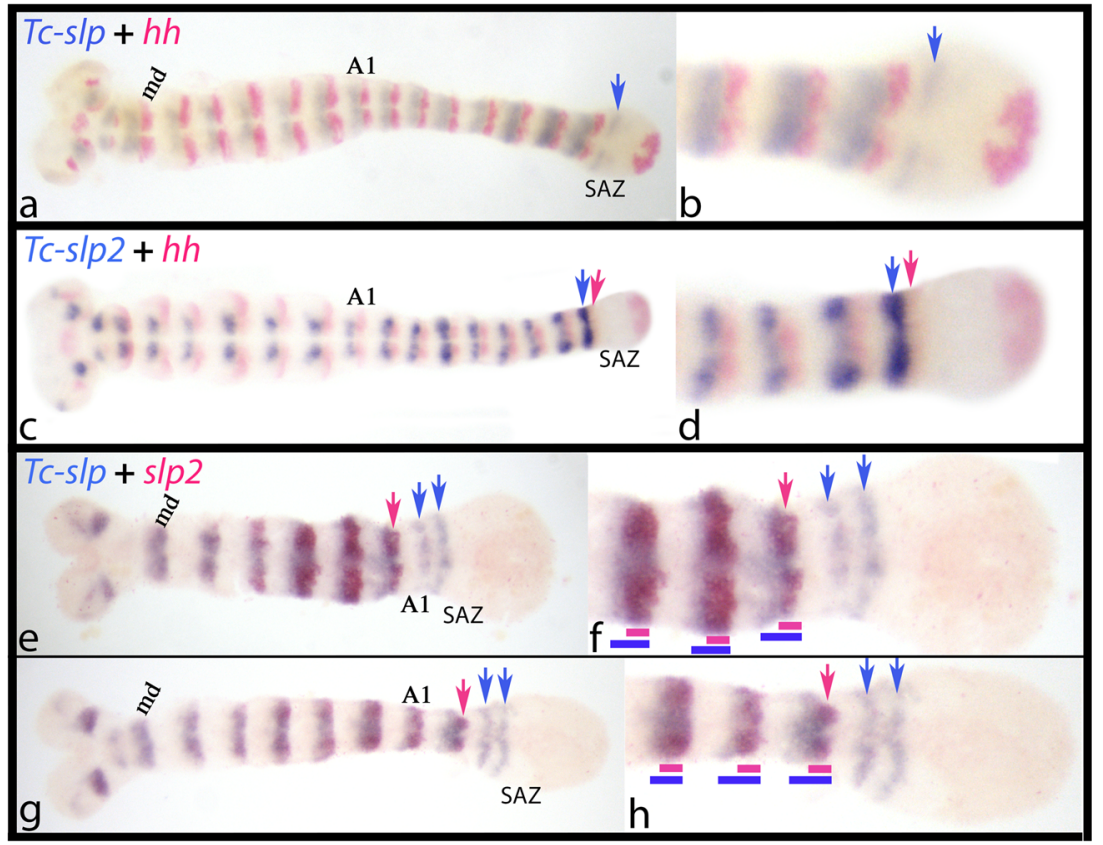

Fig. 3 Intra-segmental position of Tribolium slp and slp2. In all panels, anterior is to the left; ventral views. Panels $\mathbf{a}, \mathbf{d}, \mathbf{f}$ and $\mathbf{h}$ represent magnifications of the posterior regions of the embryos shown in $\mathbf{a}, \mathbf{c}, \mathbf{e}$ and $\mathbf{g}$ respectively. Co-expression of $\operatorname{slp}(\mathbf{a} / \mathbf{b}$, blue signal) and $\operatorname{slp} 2(\mathbf{c} / \mathbf{d}$, blue signal) with the SPG hedgehog (hh) (red signal) reveals the intrasegmental position of the two Tribolium slp genes. Both genes are expressed anterior adjacent to $h h$, and thus are co-expressed with wingless $(\mathrm{wg})$ in the posterior region of the parasegment. Note that expression of $h h$ appears after the expression of slp in the anterior of the segment

\section{Discussion}

\section{The expression profiles of the two Tribolium slp genes share common features with their Drosophila orthologs}

In Drosophila, the Sloppy-paired locus contains two copies of the gene, slp1 and slp2 (Häcker et al. 1992; Grossniklaus et al. 1992). It is therefore tempting to assume that the presence of two paralogs in Tribolium may be correlated with the function(s) of the two Drosophila slp genes, i.e. that in both species, slp1 and $\operatorname{slp} 2$, respectively, share the same function(s). In Drosophila, slp1 is expressed slightly earlier than its paralog, slp2 (Grossniklaus et al. 1992; Häcker et al. 1992). Therefore, and although the overall function of the two paralogs is largely redundant, it has been assumed that slp1 may be responsible for the early function of the Slp locus, i.e. that of a PRG, and that the later expressed $s l p 2$ may be (in concert with $s l p 1$ ) involved in the later function, i.e. that of a SPG (Cadigan et al. 1994b).

Interestingly, this situation appears to be conserved in Tribolium, where slp (the likely ortholog of Drosophila slp1) is expressed earlier than its paralog slp2. In addition, $s l p$ is expressed in a PRG-like pattern in the form of initial broad domains that later split and then correspond to addition zone (SAZ) (blue arrows in panels a and b), but that $h h$ coappears with the expression of $\operatorname{slp} 2$ (blue arrows in panels $\mathbf{c}$ and $\mathbf{d}$ ). Coexpression of $\operatorname{slp}$ (blue signal) with slp2 (red signal) (e-h) reveals that $s l p$ (blue arrows) appears earlier and more posteriorly (in the anterior SAZ) than $\operatorname{slp} 2$ (red arrows) which appears in the last newly forming segment anterior to the SAZ. Note that the expression of $s l p$ extends somewhat more anteriorly in the segments (blue bars) than slp2 (red bars). Abbreviations: A1, first abdominal segment; md, mandibular segment

expression in two adjacent segments, or indeed by intercalation (discussed below) (Fig. 2). In contrast, Tribolium slp2 expression is that of a typical SPG (cf. expression of Tribolium SPGs; e.g. Farzana and Brown 2008) with a clear single-segment periodicity (Fig. 2). The expression profiles of Tribolium slp and $s l p 2$ are thus remarkably similar to those of the two Drosophila slp genes, suggesting at least partially conserved regulation and interaction of these genes in the fly and the beetle.

\section{Pair-rule patterning: Intercalation or splitting?}

In Drosophila, the secondary PRG-stripes of slp1 and slp2 form by intercalation, i.e. de novo formation of stripes in between the first-formed primary PRG-stripes (Grossniklaus et al. 1992). In Tribolium, the situation is not that clear. It is obvious that the segmental expression of $\operatorname{slp} 2$ appears with a single-segment periodicity (this study). Posterior stripes of $s l p$, however, appear in pairs, or do they not? The mandibular (md) and maxillary (mx) stripes seem to appear as a pair (Fig. 2d) (see also Choe and Brown 2007). In the following labial (lb) segment, however, appearance of slp is delayed; this stripe intercalates (like the secondary stripes of $s l p 1$ and $s l p 2$ in Drosophila). For the next two pairs of stripes, the situation is unclear. It appears that these stripes form by splitting of an 
initially broad single domain (Fig. 2f', $\mathrm{g}^{\prime}, \mathrm{h}^{\prime}, \mathrm{i}^{\prime}$ ) (see also Choe and Brown 2007, their Fig. 2g), rather than by intercalation of the secondary (anterior) stripes. A remnant of the initial broad domain (Fig. 2f') that may document the splitting is present (Fig. 2g', arrow). Such remnant of expression has been interpreted as evidence of splitting of a PRG's initial expression in the grasshopper Schistocerca (Davis et al. 2001). The formation of stripes in the third and fourth abdominal segments (A3/A4), however, may imply intercalation (note the spacing (black bar) between the last-formed stripe in the embryo shown in Fig. $2 \mathrm{j}^{\prime}$ ). However, again, in the following stage, possible remnants of this initial domain are present between the two stripes (Fig. 2k', arrow). The situation in A7 and A8 is similar, displaying a large distance between a first-formed stripe and the stripe in A6 (Fig. $2 \mathrm{~m}^{\prime}$ ). It is therefore unclear if the secondary (anterior of each pair) stripes of $s l p$ appear by intercalation or splitting. Possibly, there are also differences in the regulation of the patterning of anterior abdominal versus posterior abdominal segments as shown for the regulation of the SPG H15 in this species (Janssen 2014).

Both splitting of initially formed broad double-segment wide stripes and intercalation of secondary stripes as seen in Drosophila appear to be a characteristic of PRG expression in general, as it is seen in a wide range of drosophilids, nondrosophilid insects with a long-germ developmental mode and in short-germ arthropods such as Tribolium, myriapods and even a mite (e.g. Binner and Sander 1997; Dearden et al. 2002; Chipman et al. 2004; Choe and Brown 2007; Wilson et al. 2010; Janssen et al. 2012). If these double-segmental patterns in the various arthropods are conserved, or have evolved independently, again, remains unclear.

\section{The intra-segmental position of slp genes in arthro- pods is conserved}

In both the beetle and the fly, both slp genes are expressed in stripes anterior adjacent to engrailed (en) and hedgehog $(h h)$, and thus, at least partly, coexpressed with wingless (wg) (Grossniklaus et al. 1992, Choe and Brown 2007, this study). The relative expression of Drosophila slp1 and slp2 in comparison with each other has not been investigated in detail, but the two slp genes are described as fully co-expressed (Grossniklaus et al. 1992, Erik Clark (personal communication)). The broader expression of $s l p$ in Tribolium compared with slp2 thus represents a difference between these two species. Data on slp expression from other insects are scarce. In another beetle, Dermestes maculatus, a single slp gene has been identified, that is expressed in a comparable pattern with that of the Tribolium slp genes, and plays a classic function as PRG (complementary to that of the other secondary PRG, paired (prd)) (Xiang et al. 2017).
Its intra-segmental position is not clear. In the true bug Oncopeltus fasciatus, the single identified slp gene is expressed as stripes with a single-segment periodicity in the anterior SAZ and newly forming segments, is under control of the bona fide gap gene giant (Liu and Patel 2010) and appears to have a conserved function as segment-polarity gene (Auman and Chipman 2018; Reding et al. 2019). Data on crustacean $s l p$ expression are not available. In the myriapods Glomeris marginata and Strigamia maritima, slp is expressed with a singlesegment periodicity in posteriorly added segments and anterior to en (Janssen et al. 2011; Green and Akam 2013). The intra-segmental position is thus conserved between insects and myriapods. Finally, in the spider Cupiennius salei, slp is expressed in stripes in newly forming segments, but its intra-segmental position is not clear (Damen et al. 2005).

\section{Possible implications for earlier studies}

The finding that Tribolium, like Drosophila, possesses two slp genes with shared and comparable (to Drosophila) expression profiles could explain some of the differences in the regulation and function of Tribolium slp reported in earlier studies, and suggests the need for a re-investigation of the function(s) of $s l p$ and slp2 in Tribolium (Choe and Brown 2007).

In Drosophila slp null mutants, expression of wg disappears from alternating segments, while in the same segments, expression of en extends towards anterior into the natural domain of $w g$ (summarized in Choe and Brown 2007). In Tribolium, however, downregulation of slp leads inter alia to defects in all anterior segments, but in posterior segments, alternating segments are affected (Choe and Brown 2007). Interestingly, however, the register in which slp affects segmentation is shifted (the alternating pattern is reversed) in these two species (Choe and Brown 2007). Another difference is that in Drosophila and the true bug Oncopeltus, but not in Tribolium, en expands towards anterior in the absence/ downregulation of Slp function (Choe and Brown 2007; Auman and Chipman 2018; Reding et al. 2019). While the former cannot easily be explained by the presence of a second paralog in Tribolium (i.e. slp2), it could be that en remains restrained to its natural domain in the absence of $s l p$ in Tribolium because slp2 acts as a potential repressor of enexpression. Alternatively, the remaining low activity of $s l p$ after RNAi may be responsible for this difference.

Funding information Open access funding provided by Uppsala University. Financial support was provided by the Swedish Natural Science Council (VR) (grant no. 621-2011-4703). Specimens of Tribolium castaneum were provided by Gregor Bucher (University of Göttingen, Germany). 
Open Access This article is licensed under a Creative Commons Attribution 4.0 International License, which permits use, sharing, adaptation, distribution and reproduction in any medium or format, as long as you give appropriate credit to the original author(s) and the source, provide a link to the Creative Commons licence, and indicate if changes were made. The images or other third party material in this article are included in the article's Creative Commons licence, unless indicated otherwise in a credit line to the material. If material is not included in the article's Creative Commons licence and your intended use is not permitted by statutory regulation or exceeds the permitted use, you will need to obtain permission directly from the copyright holder. To view a copy of this licence, visit http://creativecommons.org/licenses/by/4.0/.

\section{References}

Akam M (1987) The molecular basis for metameric pattern in the Drosophila embryo. Development 101:1-22

Aranda M, Marques-Souza H, Bayer T, Tautz D (2008) The role of the segmentation gene hairy in Tribolium. Dev Genes Evol 218:465477

Auman T, Chipman AD (2018) Growth zone segmentation in the milkweed bug Oncopeltus fasciatus sheds light on the evolution of insect segmentation. BMC Evol Biol 18:178

Binner P, Sander K (1997) Pair-rule patterning in the honeybee Apis mellifera: expression of even-skipped combines traits known from beetles and fruitfly. Dev Genes Evol 206:447-454

Bolognesi R, Fischer TD, Brown SJ (2009) Loss of Tc-arrow and canonical Wnt signaling alters posterior morphology and pair-rule gene expression in the short-germ insect, Tribolium castaneum. Dev Genes Evol 219:369-375

Brown SJ, Hilgenfeld RB, Denell RE (1994) The beetle Tribolium castaneum has a fushi tarazu homolog expressed in stripes during segmentation. Proc Natl Acad Sci U S A 91:12922-12926

Brown SJ, Parrish JK, Beeman RW, Denell RE (1997) Molecular characterization and embryonic expression of the even-skipped ortholog of Tribolium castaneum. Mech Dev 61:165-173

Cadigan KM, Grossniklaus U, Gehring WJ (1994a) Localized expression of sloppy paired protein maintains the polarity of Drosophila parasegments. Genes Dev 8:899-913

Cadigan KM, Grossniklaus U, Gehring WJ (1994b) Functional redundancy: the respective roles of the two sloppy paired genes in Drosophila segmentation. Proc Natl Acad Sci U S A 91:6324-6328

Chipman AD, Akam M (2008) The segmentation cascade in the centipede Strigamia maritima: involvement of the Notch pathway and pair-rule gene homologues. Dev Biol 319:160-169

Chipman AD, Arthur W, Akam M (2004) A double segment periodicity underlies segment generation in centipede development. Curr Biol 14:1250-1255

Choe CP, Brown SJ (2007) Evolutionary flexibility of pair-rule patterning revealed by functional analysis of secondary pair-rule genes, paired and sloppy-paired in the short-germ insect, Tribolium castaneum. Dev Biol 302:281-294

Choe CP, Brown SJ (2009) Genetic regulation of engrailed and wingless in Tribolium segmentation and the evolution of pair-rule segmentation. Dev Biol 325:482-491

Choe CP, Miller SC, Brown SJ (2006) A pair-rule gene circuit defines segments sequentially in the short-germ insect Tribolium castaneum. Proc Natl Acad Sci U S A 103:6560-6564

Clark E (2017) Dynamic patterning by the Drosophila pair-rule network reconciles long-germ and short-germ segmentation. PLoS Biol 15: e2002439
Clark E, Peel AD, Akam M (2019) Arthropod segmentation. Development 146(18):dev170480

Damen WG, Weller M, Tautz D (2000) Expression patterns of hairy, even-skipped, and runt in the spider Cupiennius salei imply that these genes were segmentation genes in a basal arthropod. Proc Natl Acad Sci U S A 97:4515-4519

Damen WG, Janssen R, Prpic NM (2005) Pair rule gene orthologs in spider segmentation. Evol Dev 7:618-628

Davis GK, Patel NH (2003) Playing by pair rules? BioEssays 25:425-429

Davis GK, Jaramillo CA, Patel NH (2001) Pax group III genes and the evolution of insect pair-rule patterning. Development 128:34453458

Dearden PK, Donly C, Grbic M (2002) Expression of pair rule gene homologues: early patterning of the two-spotted spider mite Tetranychus urticae. Development 129:5461-5472

Eckert C, Aranda M, Wolff C, Tautz D (2004) Separable stripe enhancer elements for the pair-rule gene hairy in the beetle Tribolium. EMBO 5:638-642

El-Sherif E, Averof M, Brown SJ (2012) A segmentation clock operating in blastoderm and germband stages of Tribolium development. Development 139:4341-4346

El-Sherif E, Zhu X, Fu J, Brown SJ (2014) Caudal regulates the spatiotemporal dynamics of pair-rule waves in Tribolium. PLoS Genet 10: e1004677

Erezyilmaz DF, Kelstrup HC, Riddiford LM (2009) The nuclear receptor E75A has a novel pair-rule-like function in patterning the milkweed bug, Oncopeltus fasciatus. Dev Biol 334:300-310

Farzana L, Brown SJ (2008) Hedgehog signaling pathway function conserved in Tribolium segmentation. Dev Genes Evol 218:181-192

Fröhnhofer HG, Nüsslein-Volhard C (1986) Organization of anterior pattern in the Drosophila embryo by the maternal gene bicoid. Nature 324:120-125

Green J, Akam M (2013) Evolution of the pair rule gene network: insights from a centipede. Dev Biol 382:235-245

Grossniklaus U, Pearson RK, Gehring WJ (1992) The Drosophila sloppy paired locus encodes two proteins involved in segmentation that show homology to mammalian transcription factors. Genes Dev 6: $1030-1051$

Häcker U, Grossniklaus U, Gehring WJ, Jäckle H (1992) Developmentally regulated Drosophila gene family encoding the fork head domain. Proc Natl Acad Sci U S A 89:8754-8758

Huelsenbeck JP, Ronquist F (2001) MRBAYES: bayesian inference of phylogenetic trees. Bioinformatics 17:754-755

Hughes CL, Kaufman TC (2002) Exploring myriapod segmentation: the expression patterns of even-skipped, engrailed, and wingless in a centipede. Dev Biol 247:47-61

Ingham PW, Baker NE, Martinez-Arias A (1988) Regulation of segment polarity genes in the Drosophila blastoderm by fushi tarazu and even skipped. Nature 331:73-75

Jäckle H, Hoch M, Pankratz MJ, Gerwin N, Sauer F, Brönner G (1992) Transcriptional control by Drosophila gap genes. J Cell Sci Suppl $16: 39-51$

Janssen R (2014) Gene expression suggests double-segmental and singlesegmental patterning mechanisms during posterior segment addition in the beetle Tribolium castaneum. Int J Dev Biol 58:343-347

Janssen R, Budd GE (2013) Deciphering the onychophoran 'segmentation gene cascade': gene expression reveals limited involvement of pair rule gene orthologs in segmentation, but a highly conserved segment polarity gene network. Dev Biol 382:224-234

Janssen R, Budd GE, Damen WG, Prpic NM (2008) Evidence for Wgindependent tergite boundary formation in the millipede Glomeris marginata. Dev Genes Evol 218:361-370

Janssen R, Budd GE, Prpic NM, Damen WG (2011) Expression of myriapod pair rule gene orthologs. Evodevo 2:5 
Janssen R, Damen WGM, Budd GE (2012) Expression of pair rule gene orthologs in the blastoderm of a myriapod: evidence for pair rulelike mechanisms? BMC Dev Biol 12:15

Janssen R, Andersson E, Betnér E, Bijl S, Fowler W, Höök L, Leyhr J, Mannelqvist A, Panara V, Smith K, Tiemann S (2018) Embryonic expression patterns and phylogenetic analysis of panarthropod sox genes: insight into nervous system development, segmentation and gonadogenesis. BMC Evol Biol 18:88

Liu PZ, Kaufman TC (2005) even-skipped is not a pair-rule gene but has segmental and gap-like functions in Oncopeltus fasciatus, an intermediate germband insect. Development 132:2081-2092

Liu PZ, Patel NH (2010) giant is a bona fide gap gene in the intermediate germband insect, Oncopeltus fasciatus. Development 137:835-844

Maderspacher F, Bucher G, Klingler M (1998) Pair-rule and gap gene mutants in the flour beetle Tribolium castaneum. Dev Genes Evol 208:558-568

Mito T, Kobayashi C, Sarashina I, Zhang H, Shinahara W, Miyawaki K, Shinmyo Y, Ohuchi H, Noji S (2007) even-skipped has gap-like, pair-rule-like, and segmental functions in the cricket Gryllus bimaculatus, a basal, intermediate germ insect (Orthoptera). Dev Biol 303:202-213

Notredame C, Higgins DG, Heringa J (2000) T-Coffee: a novel method for fast and accurate multiple sequence alignment. J Mol Biol 302: 205-217

Peel A (2004) The evolution of arthropod segmentation mechanisms. Bioessays 26:1108-1116

Peel AD, Schanda J, Grossmann D, Ruge F, Oberhofer G, Gilles AF, Schinko JB, Klingler M, Bucher G (2013) Tc-knirps plays different roles in the specification of antennal and mandibular parasegment boundaries and is regulated by a pair-rule gene in the beetle Tribolium castaneum. BMC Dev Biol 13:25

Reding K, Chen M, Lu Y, Cheatle Jarvela AM, Pick L (2019) Shifting roles of Drosophila pair-rule gene orthologs: segmental expression and function in the milkweed bug Oncopeltus fasciatus. Development 146:dev181453
Rosenberg MI, Brent AE, Payre F, Desplan C (2015) Dual mode of embryonic development is highlighted by expression and function of Nasonia pair-rule genes. Elife. 3:e01440

Sarrazin AF, Peel AD, Averof M (2012) A segmentation clock with two segment periodicity in insects. Science 336:338-341

Schinko J, Posnien N, Kittelmann S, Koniszewski N, Bucher G (2009) Single and double whole-mount in situ hybridization in red flour beetle (Tribolium) embryos. Cold Spring Harb Protoc 2009(8): pdb.prot5258

Schönauer A, Paese CL, Hilbrant M, Leite DJ, Schwager EE, Feitosa NM, Eibner C, Damen WG, McGregor AP (2016) The Wnt and Delta-Notch signalling pathways interact to direct pair-rule gene expression via caudal during segment addition in the spider Parasteatoda tepidariorum. Development 143:2455-2463

Schoppmeier M, Damen WG (2005) Expression of Pax group III genes suggests a single-segmental periodicity for opisthosomal segment patterning in the spider Cupiennius salei. Evol Dev 7:160-167

Schröder R, Jay DG, Tautz D (2000) Elimination of EVE protein by CALI in the short germ band insect Tribolium suggests a conserved pair-rule function for even skipped. Mech Dev 90:329

Sommer RJ, Tautz D (1993) Involvement of an orthologue of the Drosophila pair-rule gene hairy in segment formation of the short germ-band embryo of Tribolium (Coleoptera). Nature 361:448-450

Wilson MJ, Havler M, Dearden PK (2010) Giant, Krüppel, and caudal act as gap genes with extensive roles in patterning the honeybee embryo. Dev Biol 339:200-211

Xiang J, Reding K, Heffer A, Pick L (2017) Conservation and variation in pair-rule gene expression and function in the intermediate-germ beetle Dermestes maculatus. Development 144:4625-4636

Publisher's note Springer Nature remains neutral with regard to jurisdictional claims in published maps and institutional affiliations. 